Pacific

Journal of

Mathematics

FROBENIUS-SCHUR INDICATORS FOR A CLASS OF FUSION CATEGORIES

SONIA NATALE

Volume $221 \quad$ No. 2

October 2005 


\title{
FROBENIUS-SCHUR INDICATORS FOR A CLASS OF FUSION CATEGORIES
}

\author{
SONIA NATALE
}

\begin{abstract}
We give an explicit description of group-theoretical quasi-Hopf algebras up to gauge equivalence. We then use it to compute the Frobenius-Schur indicators for group-theoretical fusion categories.
\end{abstract}

\section{Introduction}

There are strong analogies between the theory of finite groups and the theory of semisimple Hopf algebras; some of them, however, still remain conjectural. In particular, the problem of classifying semisimple Hopf algebras, say over the field of complex numbers, seems to be a difficult one, even in low dimensions. Perhaps the most important feature of these objects, which relates them to other branches of mathematics and physics, is that their category of representations is a special case of a so called fusion category. This fact leads to the consideration of the classification problem, not only modulo Hopf algebra isomorphisms, but modulo gauge equivalences: roughly, two finite-dimensional (quasi)-Hopf algebras $H$ and $H^{\prime}$ give rise to the same fusion category of representations if and only if they are gauge equivalent, in the sense that $H=H^{\prime}$ as algebras, and the comultiplication of $H^{\prime}$ is obtained by "twisting" that of $H$ by means of $\Delta_{H^{\prime}}(h)=F \Delta(h) F^{-1}$, for some gauge transformation $F \in(H \otimes H)^{\times}$.

An important class of examples of semisimple quasi-Hopf algebras was introduced by Ostrik [2002] and studied by Etingof, Nikshych and Ostrik [Etingof et al. 2002]. Called group-theoretical, these are, by definition, those for which the category of representations is a group-theoretical category $\mathscr{C}(G, \omega, F, \alpha)$, where $G$ is a finite group, $F \subseteq G$ is a subgroup, $\omega: G \times G \times G \rightarrow k^{\times}$is a normalized 3cocycle and $\alpha: F \times F \rightarrow k^{\times}$is a normalized 2-cochain, such that $\left.\omega\right|_{F}=d \alpha$. More precisely, $\mathscr{C}(G, \omega, F, \alpha)$ is the category of $k_{\alpha} F$-bimodules in the tensor category $\mathrm{Vec}_{\omega}^{G}$ of finite-dimensional $G$-graded vector spaces, with associativity constraint given by $\omega$. All concrete known examples of semisimple Hopf algebras (and this

MSC2000: $16 \mathrm{~W} 30$.

Keywords: semisimple quasi-Hopf algebra, Frobenius-Schur indicators.

This work was partially supported by CONICET, CONICOR, Fundación Antorchas and Secyt (UNC). 
does not extend to the quasi-context), turn out to be group-theoretical; this can be seen as a consequence of our results in [Natale 2003]. The following question has been posed in [Etingof et al. 2002]. An answer to this question (even an affirmative one) would be of great significance in the classification program.

Question 1-1. Does there exist a semisimple Hopf algebra which is not grouptheoretical?

The categorical nature of this question leads naturally to the problem of finding and computing gauge invariants of group-theoretical quasi-Hopf algebras, that is, invariants which depend on the gauge equivalence class of the object rather than on the isomorphism class itself.

Recently, Mason and Ng have constructed a gauge invariant, the FrobeniusSchur indicators, for semisimple quasi-Hopf algebras [Mason and $\mathrm{Ng}$ 2005]. They have proved a generalization of the Frobenius-Schur Theorem for finite groups; compare [Serre 1967]. Their construction extends results of Linchenko and Montgomery for semisimple Hopf algebras [Linchenko and Montgomery 2000]; it also extends results of Bantay on the Frobenius-Schur indicators for the DijkgraafPasquier-Roche quasi-Hopf algebra $D^{\omega} G$, after the definition in [Bantay 1997] of the indicators attached to conformal field theories.

Essentially, Frobenius-Schur indicators were defined in a categorical fashion for any semisimple rigid tensor category which is pivotal, i.e., which admits a natural tensor isomorphism between the identity and the second left duality functors, in the work of Fuchs, Ganchev, Szlachányi and Vescernyés [Fuchs et al. 1999]. It is shown in [Etingof et al. 2002] that representation categories of semisimple quasiHopf algebras are in fact pivotal.

Other gauge invariants can be attached to a semisimple quasi-Hopf algebra. One of the most studied is the $K_{0}$-ring of its representation category. This invariant does not distinguish the group algebras of the two nonabelian groups of order 8: the dihedral group $D_{4}$ and the quaternionic group $Q_{2}$. However, by a result of Tambara and Yamagami [1998], these two groups are not gauge equivalent. In their paper, Mason and $\mathrm{Ng}$ have noted that the Frobenius-Schur indicators do distinguish the dihedral and quaternionic groups. As pointed out to us by the referee, in some cases it may happen that the Frobenius-Schur indicators contain less information than the $K_{0}$-ring, e.g., in the case of dual group algebras. So, in some sense, these two invariants are of very different nature.

In this paper we give an explicit description of group-theoretical Hopf algebras and use it to compute their irreducible characters and Frobenius-Schur indicators. Our main original contributions in the description of the quasi-structure are on the one hand the proof of the existence of a certain normalization of the 3-cocycle $\omega$ 
(Proposition 4.2), and on the other hand, the construction of a quasi-antipode (Theorem 4.12) which is, of course, essential in the computation of the Frobenius-Schur invariants. We obtain the following formula for the indicator of the irreducible character $\chi$ :

$$
\chi\left(v_{A^{\mathrm{op}}}\right)=|F|^{-1} \sum_{(q \triangleleft x) \cdot q=e} \omega(x q, x q, x q) \quad \chi\left(\delta_{q}(x q)^{2}\right),
$$

which involves a certain normalization of the 3-cocycle $\omega$. See Corollary 5.4. Here, $q$ runs over an appropriate choice of representatives of $G$ modulo $F$.

One instance of these examples comes from an exact factorization $G=F Q$ of the group $G$ into its subgroups $F$ and $Q$. In this case, there is a group

$$
\operatorname{Opext}\left(k F, k^{Q}\right)
$$

that classifies the abelian Hopf algebra extensions of $k^{Q}$ by $k F$ : as a Hopf algebra, the extension corresponding to an element $[\sigma, \tau] \in \operatorname{Opext}\left(k F, k^{Q}\right)$ is a bicrossed product $k^{Q} \#_{\sigma}^{\tau} k F$, where $\sigma: F \times F \rightarrow\left(k^{Q}\right)^{\times}$and $\tau: Q \times Q \rightarrow\left(k^{F}\right)^{\times}$are a pair of compatible cocycles; see [Natale 2003, Theorem 1.2]. In this case, the 3-cocycle $\omega$ in our formula for the Frobenius-Schur indicators is the one associated to $[\sigma, \tau]$ in the Kac exact sequence. This gives an alternative compact expression for the formula found by Kashina, Mason and Montgomery in [Kashina et al. 2002].

We would like to point out that the description for the quasi-Hopf algebra structure for group-theoretical quasi-Hopf algebras generalizes the construction of the twisted quantum doubles $D^{\omega} G$ by Dijkgraaf, Pasquier and Roche. This agrees with the characterization given in [Natale 2003] in terms of quantum (or Drinfeld) doubles; so in some sense these quasi-Hopf algebras are all of DPR-type.

The paper is organized as follows. In Sections 2 and 3 we recall the definition of the indicators constructed in [Mason and $\mathrm{Ng} 2005$ ] and the definition and main properties of group-theoretical categories as given in [Ostrik 2002; Etingof et al. 2002]. In Section 4 we give a description, up to gauge equivalence, of the structure of group-theoretical quasi-Hopf algebras, and finally in Section 5 we give an explicit formula for the Frobenius-Schur indicators of group-theoretical categories. We consider some examples in Section 6. Throughout this paper we work over an algebraically closed field $k$ of characteristic zero.

\section{Frobenius-Schur indicators}

Let $(H, \Delta, \epsilon, \Phi, \mathscr{Y}, \alpha, \beta)$ be a finite-dimensional semisimple quasi-Hopf algebra [Drinfeld 1989] (later on indicated $(H, \Phi)$ for short), that is, $H$ is an associative unital algebra over $k$ which is semisimple and finite-dimensional; $\epsilon: H \rightarrow k$ and 
$\Delta: H \rightarrow H \otimes H$ are algebra maps; $\Phi \in H^{\otimes 3}$ is an invertible element such that $(\mathrm{id} \otimes \mathrm{id} \otimes \Delta)(\Phi)(\Delta \otimes \mathrm{id} \otimes \mathrm{id})(\Phi)=(1 \otimes \Phi)(\mathrm{id} \otimes \Delta \otimes \mathrm{id})(\Phi)(\Phi \otimes 1)$,

$(\mathrm{id} \otimes \epsilon \otimes \mathrm{id})(\Phi)=1 \otimes 1$, $(\epsilon \otimes \mathrm{id}) \Delta(h)=h=(\mathrm{id} \otimes \epsilon) \Delta(h)$, $\Phi(\Delta \otimes \mathrm{id}) \Delta(h) \Phi^{-1}=(\mathrm{id} \otimes \Delta) \Delta(h)$,

for all $h \in H$. The map $\mathscr{S}: H \rightarrow H^{\text {op }}$ is an algebra anti-automorphism of $H$; $\alpha, \beta \in H$ are such that

$$
\mathscr{Y}\left(h_{1}\right) \alpha h_{2}=\epsilon(h) \alpha \quad \text { and } \quad h_{1} \beta \mathscr{Y}\left(h_{2}\right)=\epsilon(h) \beta \quad \text { for all } h \in H
$$

and

$$
\Phi^{(1)} \beta \mathscr{S}\left(\Phi^{(2)}\right) \alpha \Phi^{(3)}=1=\mathscr{S}\left(\Phi^{(-1)}\right) \alpha \Phi^{(-2)} \beta \mathscr{S}\left(\Phi^{(-3)}\right),
$$

where we are using the abbreviated notation $\Phi=\Phi^{(1)} \otimes \Phi^{(2)} \otimes \Phi^{(3)}$ and $\Phi^{-1}=$ $\Phi^{(-1)} \otimes \Phi^{(-2)} \otimes \Phi^{(-3)}$.

The category $\operatorname{Rep} H=: \operatorname{Rep}(H, \Phi)$ is a fusion category, in the terminology of [Etingof et al. 2002]. The associativity constraint is given by the natural action of $\Phi$; the left dual of an object $V$ of $\operatorname{Rep} H$ is the vector space $V^{*}=\operatorname{Hom}(V, k)$ with the $H$-action $\langle h . f, v\rangle=\langle f, \mathscr{Y}(h) v\rangle$; and the evaluation and coevaluation maps are given, respectively, by

$$
\begin{aligned}
\text { ev }: V^{*} \otimes V \rightarrow k, & \operatorname{ev}(f \otimes v)=\langle f, \alpha \cdot v\rangle, \\
\operatorname{coev}: k \rightarrow V \otimes V^{*}, & 1 \mapsto \sum_{i} \beta . v_{i} \otimes v^{i},
\end{aligned}
$$

for all $f \in V^{*}, v \in V$, where $\left(v_{i}\right)$ and $\left(v^{i}\right)$ are dual bases of $V$.

Two quasi-Hopf algebras $H_{1}$ and $H_{2}$ are called gauge equivalent if there exists a gauge transformation, i.e., an invertible normalized element $F \in H_{1} \otimes H_{1}$, such that $\left(H_{1}\right)_{F}$ and $H_{2}$ are isomorphic as quasi-bialgebras, where $\left(H_{1}\right)_{F}$ is the quasi-Hopf algebra $\left(H_{1}, \Delta_{F}, \epsilon, \Phi_{F}, \mathscr{Y}_{F}, \alpha_{F}, \beta_{F}\right)$ defined by

$$
\begin{gathered}
\Delta_{F}(h)=F \Delta(h) F^{-1}, \quad h \in H, \\
\Phi_{F}=(1 \otimes F)(\mathrm{id} \otimes \Delta)(F) \Phi(\Delta \otimes \mathrm{id})\left(F^{-1}\right)\left(F^{-1} \otimes 1\right), \\
\alpha_{F}=\mathscr{S}\left(F^{(-1)}\right) \alpha F^{(-2)}, \quad \beta_{F}=F^{(1)} \beta \mathscr{S}\left(F^{(2)}\right),
\end{gathered}
$$

for $F=F^{(1)} \otimes F^{(2)}$ and $F^{-1}=F^{(-1)} \otimes F^{(-2)}$.

Two finite-dimensional quasi-Hopf algebras $H_{1}$ and $H_{2}$ are gauge equivalent if and only if Rep $H_{1}$ is equivalent to Rep $H_{2}$ as $k$-linear tensor categories. See [Etingof and Gelaki 2002]. 
Remark 2.1. It is shown in [Etingof et al. 2002] that the fusion categories of the form $\operatorname{Rep}(H, \Phi)$ are exactly those for which the Frobenius-Perron dimensions of simple objects are integers.

Now let $(H, \Phi)$ be a finite-dimensional quasi-Hopf algebra. A normalized twosided integral of $H$ is an element $\Lambda \in H$ such that

$$
h \Lambda=\epsilon(h) \Lambda=\Lambda h \quad \text { for all } h \in H
$$

and $\epsilon(\Lambda)=1$. Suppose that $(H, \Phi)$ is semisimple. Then $H$ contains a unique normalized two-sided integral [Hausser and Nill 1999].

The following definition is due to Mason and $\mathrm{Ng}$ [2005]. It generalizes a previous definition for semisimple Hopf algebras given by Linchenko and Montgomery [2000].

Definition 2.2. Let $(H, \Phi)$ be a finite-dimensional semisimple quasi-Hopf algebra and let $\Lambda \in H$ be a normalized two-sided integral. Let also $\chi \in H^{*}$ be an irreducible character of $H$. The Frobenius-Schur indicator of $\chi$ is the element $\chi\left(v_{H}\right)$, where $v_{H}$ is the canonical central element of $H$ given by

$$
v_{H}=m\left(q_{L} \Delta(\Lambda) p_{L}\right)
$$

here, $m: H \otimes H \rightarrow H$ is the multiplication map, and $q_{L}, p_{L} \in H^{\otimes 2}$ are defined by

$$
q_{L}:=\mathscr{Y}\left(\Phi^{(-1)}\right) \alpha \Phi^{(-2)} \otimes \Phi^{(-3)}, \quad p_{L}:=\Phi^{(2)} \mathscr{Y}^{-1}\left(\Phi^{(1)} \beta\right) \otimes \Phi^{(3)} .
$$

The family of Frobenius-Schur indicators $\left\{\chi\left(v_{H}\right)\right\}_{\chi}$ is an invariant of the $k$ linear tensor category $\operatorname{Rep}(H, \Phi)$. This means that it is invariant under gauge transformations of $(H, \Phi)$.

Also, if $\alpha$ and $\beta$ are invertible elements of $H$, then the canonical central element $v_{H}$ can be computed as follows [Mason and $\mathrm{Ng}$ 2005, Corollary 3.5]:

$$
v_{H}=\left(\Lambda_{1} \Lambda_{2}\right)(\beta \alpha)^{-1}=(\beta \alpha)^{-1}\left(\Lambda_{1} \Lambda_{2}\right) .
$$

In analogy with finite group situation, the Frobenius-Schur indicator of the irreducible character $\chi=\chi_{V}$ satisfies the following:

(i) $\chi\left(v_{H}\right)=0,1$ or -1 , and $\chi\left(v_{H}\right) \neq 0$ if and only if $\chi=\chi^{*}$

(ii) $\chi\left(v_{H}\right)=1$ (respectively -1 ) if and only if $V$ admits a nondegenerate bilinear form $\langle\rangle:, V \otimes V \rightarrow k$, with adjoint $\mathscr{Y}$, such that $\langle x, y\rangle=\left\langle y, g^{-1} x\right\rangle$ (respectively, $\left.\langle x, y\rangle=-\left\langle y, g^{-1} x\right\rangle\right)$, where $g \in H$ is the so called trace element of $H$.

\section{Group theoretical fusion categories}

Group theoretical categories were introduced in [Ostrik 2002, Section 3] and also studied in [Etingof et al. 2002]. In this section we recall their definition and basic properties. 
Let $G$ be a finite group, and let $F \subseteq G$ be a subgroup. The identity element of $G$ will be denoted by $e$. Let the following data be given:

- a normalized 3-cocycle $\omega: G \times G \times G \rightarrow k^{\times}$, meaning that $\omega$ satisfies

$$
\omega(a b, c, d) \omega(a, b, c d)=\omega(a, b, c) \omega(a, b c, d) \omega(b, c, d)
$$

and $\omega(e, a, b)=\omega(a, e, b)=\omega(a, b, e)=1$ for all $a, b, c, d \in G$, and

- a normalized 2-cochain $\alpha: F \times F \rightarrow k^{\times}$,

all subject to the condition

$$
\left.\omega\right|_{F \times F \times F}=d \alpha .
$$

Consider the category $\operatorname{Vec}_{\omega}^{G}$ of finite-dimensional $G$-graded vector spaces, with associativity constraint given by $\omega$ : explicitly, for any three objects $U, U^{\prime}$ and $U^{\prime \prime}$ of $\operatorname{Vec}_{\omega}^{G}$, we have $a_{U, U^{\prime}, U^{\prime \prime}}:\left(U \otimes U^{\prime}\right) \otimes U^{\prime \prime} \rightarrow U \otimes\left(U^{\prime} \otimes U^{\prime \prime}\right)$, given by

$$
a_{U, U^{\prime}, U^{\prime \prime}}\left(\left(u \otimes u^{\prime}\right) \otimes u^{\prime \prime}\right)=\omega\left(\|u\|,\left\|u^{\prime}\right\|,\left\|u^{\prime \prime}\right\|\right) u \otimes\left(u^{\prime} \otimes u^{\prime \prime}\right),
$$

on homogeneous elements $u \in U, u^{\prime} \in U^{\prime}, u^{\prime \prime} \in U^{\prime \prime}$, where we use \| \| to denote degree of homogeneity. In other words, $\operatorname{Vec}_{\omega}^{G}$ is the category of representations of the quasi-Hopf algebra $k^{G}$, with associator $\omega \in\left(k^{G}\right)^{\otimes 3}$.

By (3-2), the twisted group algebra $k_{\alpha} F$ is an (associative unital) algebra in $\operatorname{Vec}_{\omega}^{G}$, and one may naturally attach to it a monoidal category. Precisely, the category $\mathscr{C}(G, \omega, F, \alpha)$ is by definition the $k$-linear monoidal category of $k_{\alpha} F$ bimodules in $\operatorname{Vec}_{\omega}^{G}$ : the tensor product is $\otimes_{k_{\alpha} F}$ and the unit object is $k_{\alpha} F$. This is a fusion category over $k$ with the property that the Frobenius-Perron dimensions of its objects are integers [Etingof et al. 2002, 8.8].

Categories of the form $\mathscr{C}(G, \omega, F, \alpha)$ are called group-theoretical [Etingof et al. 2002, Definition 8.46]. By extension, a (quasi)-Hopf algebra $A$ is called grouptheoretical if the category $\operatorname{Rep} A$ of its finite-dimensional representations is grouptheoretical.

Now let $\eta: G \times G \rightarrow k^{\times}$and $\chi: F \rightarrow k^{\times}$be normalized cochains, and let $\widetilde{\omega}: G \times G \times G \rightarrow k^{\times}, \widetilde{\alpha}: F \times F \rightarrow k^{\times}$be given by

$$
\tilde{\omega}=\omega(d \eta), \quad \tilde{\alpha}=\alpha\left(\left.\eta\right|_{F \times F}\right)(d \chi) .
$$

Then the categories $\mathscr{C}(G, \omega, F, \alpha)$ and $\mathscr{C}(G, \widetilde{\omega}, F, \widetilde{\alpha})$ are equivalent [Etingof et al. 2002, Remark 8.39].

Remark 3.1. Let $G, F, \omega$ and $\alpha$ be as above. Let $Q$ be a set of representatives of the left cosets of $F$ in $G$ such that $e \in Q$; so that every element $g \in G$ writes uniquely 
in the form $g=x p$, with $p \in Q, x \in F$. Consider the 2-cochain $\eta: G \times G \rightarrow k^{\times}$ defined in the form

$$
\eta(x p, y q):=\alpha^{-1}(x, y), \quad p, q \in Q, \quad x, y \in F .
$$

Then, taking $\chi=1$, we obtain $\widetilde{\alpha}=1$. Therefore the categories $\mathscr{C}(G, \omega, F, \alpha)$ and $\mathscr{C}(G, \widetilde{\omega}, F, 1)$ are equivalent, where $\widetilde{\omega}=\omega(d \eta)$. That is, up to monoidal equivalence, we may always assume that $\alpha=1$.

Note also that the categories $\mathscr{C}(G, \omega, F, 1)$ and $\mathscr{C}(G, \omega(d \eta), F, 1)$ are tensor equivalent for every normalized 2-cochain $\eta: G \times G \rightarrow k^{\times}$such that $\left.\eta\right|_{F \times F}$ is a coboundary.

The fiber functors $\mathscr{b}(G, \omega, F, \alpha) \rightarrow$ Vec, in the case they exist, are classified by conjugacy classes of subgroups $\Gamma$ of $G$, endowed with a 2-cocycle $\beta \in$ $Z^{2}\left(\Gamma, k^{\times}\right)$, such that the class of $\left.\omega\right|_{\Gamma}$ is trivial; $G=F \Gamma$ and the class of the cocycle $\left.\left.\alpha\right|_{F \cap \Gamma} \beta^{-1}\right|_{F \cap \Gamma}$ is nondegenerate [Ostrik 2002, Corollary 3.1].

Remark 3.2. The category $\mathscr{C}=\mathscr{C}(G, \omega, F, \alpha)$ has the property that the FrobeniusPerron dimensions of its objects are integers. A Tannaka-Krein reconstruction argument shows that $\mathscr{C}$ is equivalent to the category of representations of a semisimple quasi-Hopf algebra over $k$ [Etingof et al. 2002, Theorem 8.33].

It follows from [Etingof et al. 2002, 8.8] that duals, opposites, quotient categories, full subcategories, and tensor products of group-theoretical categories are also group-theoretical. Also, by [Etingof et al. 2002, Remark 8.47], the Drinfeld center $\mathscr{L}(\mathscr{C})$ is group-theoretical if and only if so is $\mathscr{C}$.

However, in [Etingof et al. 2002, Remark 8.48], the authors note that there exist semisimple quasi-Hopf algebras such that their category of representations are not group theoretical: an explicit example is quoted there which comes from the construction of Tambara and Yamagami [1998]. The answer to the corresponding question for semisimple Hopf algebras is unknown.

\section{Group theoretical quasi-Hopf algebras}

The aim of this section is to give an explicit description, up to gauge equivalence, of the structure of group-theoretical quasi-Hopf algebras. This will enable us to explicitly compute the Frobenius-Schur indicators of group-theoretical categories in the next section. The description is based on a result of Schauenburg [2002a, 3.4], which reconstructs a quasi-bialgebra structure from certain monoidal categories of bimodules in a more general context.

Our main new result concerning this description is the explicit construction of the quasi-antipode in the group-theoretical case, which is relevant for our purposes; see Theorem 4.12. 
An instance of this quasi-Hopf algebra construction, for the case where $\omega=1$ and $\alpha=1$, was studied in [Zhu 2001]. This case was also studied in [Beggs 2003; Beggs and Al-Shomrani 2003], from the point of view of the tensor categories of representations. We shall adopt throughout the notation in [Beggs 2003].

In what follows we shall fix a finite group $G$ and a subgroup $F \subseteq G$. Following [Zhu 2001], we shall also fix a set of simultaneous representatives of the left and right cosets of $F$ in $G, Q \subseteq G$; this is possible since $G$ is finite. Thus every element $g \in G$ has unique factorizations $g=x q=p y$, where $x, y \in F, q, p \in Q$. We assume that $e \in Q$.

The uniqueness of the factorization $G=F Q$ implies that there are well defined maps

$$
\begin{array}{ll}
\triangleright: Q \times F \rightarrow F, & \triangleleft: Q \times F \rightarrow Q, \\
\therefore Q \times Q \rightarrow Q, & \theta: Q \times Q \rightarrow F,
\end{array}
$$

determined by the conditions

$$
\begin{array}{ll}
q x=(q \triangleright x)(q \triangleleft x), & q \in Q, x \in F ; \\
p q=\theta(p, q) p . q, & p, q \in Q .
\end{array}
$$

Lemma 4.1 [Beggs 2003, Proposition 2.4]. The following identities hold, for all $p, q, r \in Q, x, y \in G$ :

(i) $p \triangleleft x y=(p \triangleleft x) \triangleleft y, p \triangleleft e=p$.

(ii) $(p . q) \triangleleft x=(p \triangleleft(q \triangleright x)) .(q \triangleleft x)$.

(iii) $p \triangleright(q \triangleright x)=\theta(p, q)((p . q) \triangleright x) \theta\left((p \triangleleft(q \triangleright x), q \triangleleft x)^{-1}, e \triangleright x=x\right.$.

(iv) $p \triangleright x y=(p \triangleright x)((p \triangleleft x) \triangleright y)$.

(v) $\theta(p, q) \theta(p . q, r)=(p \triangleright \theta(q, r)) \theta(p \triangleleft \theta(q, r), q . r)$.

(vi) $(p \triangleleft \theta(q, r)) .(q . r)=(p \cdot q) . r$.

(vii) $\theta(p, e)=\theta(e, p)=e$.

Let $\omega: G \times G \times G \rightarrow k^{\times}$be a normalized 3-cocycle such that $\left.\omega\right|_{F \times F \times F}$ is trivial. In what follows we shall fix the group-theoretical category $\mathscr{C}=\mathscr{C}(G, \omega, F, 1)$. Thus the cochain $\alpha: F \times F \rightarrow k^{\times}$of page 358 will be trivial. This is, up to monoidal equivalence, no loss of generality thanks to Remark 3.1.

Proposition 4.2. There exists a normalized 2-cochain $\eta: G \times G \rightarrow k^{\times}$such that $\left.\eta\right|_{F \times F}=1$ and $\left.\omega(d \eta)\right|_{F \times G \times G}=1=\left.\omega(d \eta)\right|_{F \times F \times Q}$.

Proof. Recall that the coboundary $d \eta: G \times G \times G \rightarrow k^{\times}$is given by

$$
(d \eta)(a, b, c)=\eta(a b, c) \eta(a, b) \eta(b, c)^{-1} \eta(a, b c)^{-1},
$$

for all $a, b, c \in G$. 
The proof will be done in three steps. First let $\eta_{1}: G \times G \rightarrow k^{\times}$be the normalized cochain given by

$$
\eta_{1}(x p, y q):=\omega(x, y, q), \quad x, y \in F, \quad p, q \in Q .
$$

Then we have $\left.\eta_{1}\right|_{G \times F}=1$ and for all $x, y, z \in F, q \in Q$, we have

$$
\begin{aligned}
\left(d \eta_{1}\right)(x, y, z q) & =\eta_{1}(x y, z q) \eta_{1}(x, y) \eta_{1}(y, z q)^{-1} \eta_{1}(x, y z q)^{-1} \\
& =\omega(x y, z, q) \omega(y, z, q)^{-1} \omega(x, y z, q)^{-1} \omega(x, y, z)^{-1} \\
& =\omega(x, y, z q)^{-1},
\end{aligned}
$$

the second equality holding because $\left.\omega\right|_{F \times F \times F}=1$. Thus $\left.\omega\left(d \eta_{1}\right)\right|_{F \times F \times G}=1$.

Put now $\omega_{0}=\omega\left(d \eta_{1}\right)$ and define $\eta_{2}: G \times G \rightarrow k^{\times}$in the form

$$
\eta_{2}(x p, y q):=\omega_{0}(x, p, y q) \omega_{0}(p, y, q)^{-1}, \quad x, y \in F, \quad p, q \in Q .
$$

Then $\left.\eta_{2}\right|_{F \times G}=1$ and we have

$$
\begin{aligned}
\left(d \eta_{2}\right)(x, y p, z q) & =\eta_{2}(x y p, z q) \eta_{2}(x, y p) \eta_{2}(y p, z q)^{-1} \eta_{2}(x, y p z q)^{-1} \\
& =\omega_{0}(x y, p, z q) \omega_{0}(p, z, q) \omega_{0}(p, z, q)^{-1} \omega_{0}(y, p, z q)^{-1} \\
& =\omega_{0}(x, y p, z q) \omega_{0}(x, y, p) \omega_{0}(x, y, p z q)^{-1} \\
& =\omega_{0}(x, y p, z q),
\end{aligned}
$$

for all $x, y, z \in F, p, q \in Q$, where in the third and fourth equalities we have used that $\left.\omega_{0}\right|_{F \times F \times G}=1$. Hence $\left.\omega_{0}\left(d \eta_{2}^{-1}\right)\right|_{F \times G \times G}=1$.

Finally, let $\omega_{1}=\omega_{0}\left(d \eta_{2}^{-1}\right)$. The condition $\left.\omega_{1}\right|_{F \times G \times G}=1$ is equivalent to $\omega_{1}(x t, g, h)=\omega_{1}(t, g, h)$, for all $x \in F, t, g, h \in G$. Hence

$$
\omega_{1}(z p, x, y q)=\omega_{1}(p, x, y) \omega_{1}(p, x y, q) \omega_{1}(p x, y, q)^{-1},
$$

for all $z \in F$.

Let $\eta_{3}: G \times G \rightarrow k^{\times}$be defined by

$$
\eta_{3}(x p, y q):=\omega_{1}(x p, y, q), \quad x, y \in F, \quad p, q \in Q .
$$

Then $\left.\eta_{3}\right|_{F \times G}=\left.\eta_{3}\right|_{G \times F}=1$, and for all $x, y \in F, p, q \in Q$,

$$
\begin{aligned}
\left(d \eta_{3}\right)(p, x, y q) & =\eta_{3}(p x, y q) \eta_{3}(p, x) \eta_{3}(x, y q)^{-1} \eta_{3}(p, x y q)^{-1} \\
& =\eta_{3}(p x, y q) \eta_{3}(p, x y q)^{-1} \\
& =\omega_{1}(p x, y, q) \omega_{1}(p, x y, q)^{-1} \\
& =\omega_{1}(p, x, y) \omega_{1}(p, x, y q)^{-1} .
\end{aligned}
$$


In particular, $\left(d \eta_{3}\right)(p, x, y)=1$, and thus

$$
\omega_{1}\left(d \eta_{3}\right)(p, x, y q)=\omega_{1}\left(d \eta_{3}\right)(p, x, y) .
$$

We claim that $\left.\omega_{1}\left(d \eta_{3}\right)\right|_{F \times G \times G}=1$. To see this, let $x, y, z \in F, p, q \in Q$. Using the equality $\left.\omega_{1}\right|_{F \times G \times G}=1$, we compute

$$
\begin{aligned}
\left(d \eta_{3}\right)(x, y p, z q) & =\eta_{3}(x y p, z q) \eta_{3}(x, y p) \eta_{3}(y p, z q)^{-1} \eta_{3}(x, y p z q)^{-1} \\
& =\omega_{1}(x y p, z, q) \omega_{1}(y p, z, q)^{-1} \\
& =\omega_{1}(p, z, q) \omega_{1}(p, z, q)^{-1}=1,
\end{aligned}
$$

which proves the claim.

In view of this, equation (4-1) is equivalent to $\left.\omega_{1}\left(d \eta_{3}\right)\right|_{G \times F \times Q}=1$. This implies the proposition, since by construction $\omega_{1}\left(d \eta_{3}\right)=\omega(d \eta)$, for a suitable normalized 2-cochain such that $\left.\eta\right|_{F \times F}=1$.

By Remark 3.1, the property $\left.\eta\right|_{F \times F}=1$ in Proposition 4.2 implies that $\mathscr{C}(G, \omega, F, 1)$ is tensor equivalent to $\mathscr{C}(G, \omega(d \eta), F, 1)$. Then we may and shall assume in what follows that the 3-cocycle $\omega: G \times G \times G \rightarrow k^{\times}$satisfies the normalization conditions

$$
\begin{aligned}
& \left.\omega\right|_{F \times G \times G}=1, \\
& \left.\omega\right|_{G \times F \times Q}=1 .
\end{aligned}
$$

These conditions are necessary in order to apply the results of [Schauenburg 2002a, 3.4]; see Definition 3.3.2 in the same paper.

Lemma 4.3. Let $g, h \in G, x, y \in F, p, q \in Q$.

(i) $\omega(x p, g, h)=\omega(p, g, h)$.

(ii) $\omega(g, y, x p)=\omega(g, y, x)$.

(iii) $\omega(g, x, p q)=\omega(g, x, \theta(p, q))$.

(iv) $\omega(p q, g, h)=\omega(p . q, g, h)$.

Proof. Parts (i) and (ii) follow from the cocycle condition (3-1) and the normalization conditions (4-2) and (4-3). Parts (iii) and (iv) are a consequence of parts (i) and (ii), respectively.

Let $A=k^{Q_{\#_{\sigma}}} k F$ be the crossed product corresponding to the action $\rightarrow: k F \otimes$ $k^{Q} \rightarrow k^{Q}$ given by

$$
(x \rightarrow f)(p)=f(p \triangleleft x) \quad \text { for } x \in F, f \in k^{Q}, p \in Q
$$

and the invertible map $\sigma: F \times F \rightarrow\left(k^{Q}\right)^{\times}$defined by $\sigma(x, y)=\sum_{p \in Q} \sigma_{p}(x, y) \delta_{p}$ for $x, y \in F$, where

$$
\sigma_{p}(x, y)=\omega(p, x, y) \quad \text { for } x, y \in F, p \in Q .
$$


The normalized 3-cocycle condition (3-1) and the normalization assumption (4-2) imply the following normalized 2-cocycle condition for $\sigma$ :

$$
\begin{gathered}
\sigma_{p \triangleleft x}(y, z) \sigma_{p}(x, y z)=\sigma_{p}(x y, z) \sigma_{p}(x, y), \\
\sigma_{e}(x, y)=\sigma_{p}(e, y)=\sigma_{p}(x, e)=1,
\end{gathered}
$$

for all $x, y, z \in F, p \in Q$. Thus $A$ is an associative algebra with unit element $\sum_{p \in Q} \delta_{p} \otimes 1$. For $f \in k^{Q}, x \in F$, the element $f \otimes x \in A$ will be denoted by $f x$. Hence, for all $x, y \in F, p, q \in Q$, we have $\left(\delta_{p} x\right) .\left(\delta_{q} y\right):=\delta_{p \triangleleft x, q} \sigma_{p}(x, y) \delta_{p} x y$.

Consider the (nonassociative) crossed product coalgebra structure on $A$ corresponding to the action $\triangleright$ and the invertible normalized map $\tau: Q \times Q \rightarrow\left(k^{F}\right)^{\times}$ defined by $\tau(p, q)=\sum_{x \in F} \tau_{x}(p, q) \delta_{x}$, where

$$
\tau_{x}(p, q)=\omega(p, q, x), \quad x \in F, p, q \in Q .
$$

Using (4-2) and again (3-1), we find that $\tau$ satisfies the "twisted" normalized 2cocycle condition

$$
\begin{array}{r}
\tau_{x}(p \triangleleft \theta(q, t), q . t) \tau_{x}(q, t) \omega(p, q, t) \sigma_{p}(\theta(q, t), q . t \triangleright x) \sigma_{p}(q \triangleright(t \triangleright x), \theta(q \triangleleft(t \triangleright x), t \triangleleft x))^{-1} \\
=\tau_{x}(p . q, t) \tau_{t \triangleright x}(p, q) \omega(p \triangleleft(q \triangleright(t \triangleright x)), q \triangleleft(t \triangleright x), t \triangleleft x)
\end{array}
$$

and $\tau_{e}(p, q)=\tau_{x}(e, q)=\tau_{x}(p, e)=1$, for all $x \in F, p, q, t \in Q$.

Explicitly, we have

$$
\Delta\left(\delta_{p} x\right):=\sum_{s . t=p} \tau_{x}(s, t) \delta_{s}(t \triangleright x) \otimes \delta_{t} x, \quad p \in Q, x \in F .
$$

The counit for this coalgebra is $\epsilon \otimes \epsilon$.

Theorem 4.4. These algebra and coalgebra structures combine into a quasibialgebra structure on $A^{\mathrm{op}}$ with associator $\Phi \in A^{\otimes 3}$ given by

$$
\Phi=\sum_{p, q, r \in Q} \omega(p, q, r) \delta_{p} \theta(q, r) \otimes \delta_{q} \otimes \delta_{r} .
$$

There is a monoidal equivalence $\operatorname{Rep}\left(A^{\mathrm{op}}, \Phi\right) \sim \mathscr{C}(G, F, \omega, 1)$.

Note that $\Phi$ is invertible, with inverse

$$
\Phi^{-1}=\sum_{p, q, r \in Q} \omega(p, q, r)^{-1} \sigma_{p}\left(\theta(q, r), \theta(q, r)^{-1}\right)^{-1} \delta_{p \triangleleft \theta(q, r)} \theta(q, r)^{-1} \otimes \delta_{q} \otimes \delta_{r} .
$$

Proof. Our definitions are dual to the ones in [Schauenburg 2002a, 3.4.2, 3.4.5]; with the conventions of that paper, $\Phi$ is replaced by $\Phi^{-1}$ in condition (2-1). Thus $A^{\text {op }}$ is a quasi-bialgebra. The monoidal equivalence $\operatorname{Rep}\left(A^{\mathrm{op}}, \Phi\right) \sim \mathscr{C}(G, F, \omega, 1)$ follows from Corollary 3.4.4 in the same paper. 
Since $\mathscr{C}(G, \omega, F, 1)$ is a rigid tensor category, it follows from [Schauenburg $2002 \mathrm{~b}]$ that $A^{\text {op }}$ is a quasi-Hopf algebra. We shall give the quasi-antipode in the next subsection.

By Remark 3.1, every group-theoretical category is equivalent to one of the form $\mathscr{C}(G, \omega, F, 1)$, for suitable $G, F$ and $\omega$, where $\omega$ satisfies (4-2), (4-3), in view of Proposition 4.2. This gives us the following theorem.

Theorem 4.5. Let $(H, \phi)$ be a finite-dimensional quasi-Hopf algebra. Then $(H, \phi)$ is group-theoretical if and only if it is gauge equivalent to a quasi-Hopf algebra of the form $\left(A^{\mathrm{op}}, \Phi\right)$, associated to suitable data $G, F, Q$ and $\omega$ satisfying (4-2) and (4-3).

We shall use the symbol $\circ$ to denote the multiplication in $A^{\mathrm{op}}$, so that $a \circ b=b . a$ for all $a, b \in A^{\text {op }}$.

Remark 4.6. Using the properties listed in Lemma 4.1 and the normalization conditions (4-2) and (4-3), it is not difficult to check that $A^{\mathrm{op}}$ is a quasi-bialgebra. For instance, $\Delta: A \otimes A \rightarrow A$ is an algebra map because of parts (ii) and (iv) of Lemma 4.1 and the following relationship between $\sigma$ and $\tau$ :

(4-6) $\sigma_{t . s}(x, y) \tau_{x y}(t, s)=\tau_{x}(t, s) \tau_{y}(t \triangleleft(s \triangleright x), s \triangleleft x) \sigma_{t}(s \triangleright x,(s \triangleleft x) \triangleright y) \sigma_{s}(x, y)$,

for all $s, t \in Q, x, y \in F$, which is a consequence of (4-3) and (3-1). Compare with [Masuoka 1999, Proposition 4.7].

Remark 4.7. Identify $\sigma$ and $\tau$ with maps

$$
\sigma: Q \times F \times F \rightarrow k^{\times}, \quad \tau: Q \times Q \times F \rightarrow k^{\times} .
$$

Then the tuple

$$
\left(\Delta_{G}, 1,1, .: Q \times Q \rightarrow Q, \triangleleft, \triangleright, \theta,\left.\omega\right|_{Q \times Q \times Q}, \tau, \sigma\right)
$$

constitutes the skeleton of $(k G, \omega)$ [Schauenburg 2002a, Definition 4.1.1].

We now give the construction of a quasi-antipode for $A^{\text {op }}$. We shall need the existence of inverses for the (nonassociative) multiplication in $Q$. This is guaranteed by the next lemma.

Lemma 4.8. The set $Q$ has well-defined left and right inverses with respect to the multiplication .; that is, for every $p \in Q$ there exist unique $p^{L}, p^{R} \in Q$ such that $p^{L} \cdot p=e=p \cdot p^{R}$.

(By definition, $p p^{R}=\theta\left(p, p^{R}\right)$ and $p^{L} p=\theta\left(p^{L}, p\right)$.)

Proof. As to left inverses, the lemma is contained in [Beggs 2003, Proposition 2.3]. To prove the statement concerning right inverses, we shall use the assumption that $Q$ is also a set of representatives of the left cosets of $F$ in $G$. 
Let $p \in Q$. Since the factorization $G=Q F$ is exact, there exist unique $s \in Q$, $x \in F$, such that $p^{-1}=s x$. Then $e=p p^{-1}=p s x=\theta(p, s)(p . s) x$; thus

$$
\theta(p, s)^{-1}=(p . s) x .
$$

Because $p . s \in Q$ and $\theta(p, s)^{-1}, x \in F$, the exactness of the factorization $G=Q F$ implies that p.s $=e$.

We now show the uniqueness of such $s$, which gives the statement with $s=p^{R}$. Suppose that $s^{\prime} \in Q$ is such that $p . s^{\prime}=e$. Then $p s^{\prime}, p s \in F$, and therefore also $\left(s^{\prime}\right)^{-1} s=\left(p s^{\prime}\right)^{-1} p s \in F$. This implies that $s^{\prime}=s$, whence the uniqueness.

For later use, we give in the next lemma some of the relations between ()$^{L}$, ()$^{R}$ and the actions $\triangleright, \triangleleft$. The content of the lemma is part of [Beggs 2003, Section 4].

Lemma 4.9. The following relations hold, for all $p \in Q$ :

(i) $p^{-1}=\theta\left(p^{L}, p\right)^{-1} p^{L}=p^{R} \theta\left(p, p^{R}\right)^{-1}$.

(ii) $p^{L} \triangleleft \theta\left(p, p^{R}\right)=p^{R}$ and $p^{L} \triangleright \theta\left(p, p^{R}\right)=\theta\left(p^{L}, p\right)$.

(iii) $p^{L L}=p \triangleleft \theta\left(p^{L}, p\right)^{-1}$.

(iv) $(p \triangleleft x)^{L}=p^{L} \triangleleft(p \triangleright x)$.

Proof. This follows from the definitions and Lemma 4.1.

Definition 4.10. We introduce for notational convenience the map $\succ: Q \times F \rightarrow F$ defined by

$$
p \succ x=\theta\left(p, p^{R}\right)^{-1}(p \triangleright x) \theta\left(p \triangleleft x,(p \triangleleft x)^{R}\right),
$$

for all $p \in Q, x \in F$.

Lemma 4.11. Let $p \in Q$ and $x, y \in F$.

(i) $p \succ\left(p^{R} \triangleright x\right)=x=p^{R} \triangleright(p \succ x)$.

(ii) $(p \triangleleft x)^{R} \triangleleft(p \succ x)^{-1}=p^{R}$.

(iii) $p \succ(x y)=(p \succ x)((p \triangleleft x) \succ y)$.

(iv) $p \succ \theta\left(p^{L}, p\right)^{-1}=\theta\left(p, p^{R}\right)^{-1}$.

In particular, for all $p \in Q$ the map $p \succ \ldots: F \rightarrow F$ is bijective, the inverse being $p^{R} \triangleright{ }_{-}: F \rightarrow F$.

Proof. We shall prove part (iv), the proof of (i)-(iii) being straightforward. By Lemma 4.9(iii), we have $p \theta\left(p^{L}, p\right)^{-1}=\left(p \triangleright \theta\left(p^{L}, p\right)^{-1}\right) p^{L L}$, and on the other hand, $p \theta\left(p^{L}, p\right)^{-1}=\left(p^{L}\right)^{-1}=\theta\left(p^{L L}, p^{L}\right)^{-1} p^{L L}$.

By the exactness of the factorization $G=F Q$, we get

$$
\theta\left(p^{L L}, p^{L}\right)^{-1}=p \triangleright \theta\left(p^{L}, p\right)^{-1} .
$$


From this and the definitions, we get

$$
p \succ \theta\left(p^{L}, p\right)^{-1}=\theta\left(p, p^{R}\right)^{-1}\left(p \triangleright \theta\left(p^{L}, p\right)^{-1}\right) \theta\left(p^{L L}, p^{L}\right)=\theta\left(p, p^{R}\right)^{-1}
$$

Theorem 4.12. There is a quasi-Hopf algebra structure on $A^{\mathrm{op}}$ with quasi-antipode $\mathscr{S}: A^{\mathrm{op}} \rightarrow A^{\mathrm{op}}$ given by

$$
\mathscr{S}\left(\delta_{p} x\right)=\tau_{p \succ x}\left(p, p^{R}\right)^{-1} \sigma_{p^{R}}\left(p \succ x,(p \succ x)^{-1}\right)^{-1} \delta_{(p \triangleleft x)^{R}}(p \succ x)^{-1} .
$$

We have $\alpha=1$ and

$$
\beta=\sum_{q \in Q} \omega\left(q^{-1}, q, q^{-1}\right) \delta_{q} \theta\left(q^{L}, q\right)^{-1}=\sum_{q \in Q} \omega\left(q, q^{-1}, q\right)^{-1} \delta_{q} \theta\left(q^{L}, q\right)^{-1} .
$$

Compare with the formulas given in [Zhu 2001; Beggs 2003] for the case where $\omega=1$.

Proof. We use freely the relations in Lemma 4.3 and the normalization conditions (4-2) and (4-3). Using relation (4-6) and Lemma 4.11, it is straightforward to see that $\mathscr{S}$ is an anti-algebra map. The injectivity of $\mathscr{S}$ follows from the injectivity of the map $p \succ \ldots: F \rightarrow F$ and from Lemma 4.11(ii). Therefore $\mathscr{Y}: A^{\mathrm{op}} \rightarrow A^{\mathrm{op}}$ is an algebra anti-automorphism.

We now check condition (2-2). Let $p \in Q, x \in F$, and let $X=\delta_{p} x \in A^{\text {op }}$. Then

$$
\begin{aligned}
& \mathscr{Y}\left(X_{1}\right) \circ \alpha \circ X_{2}=\mathscr{Y}\left(X_{1}\right) \circ X_{2}=X_{2} . \mathscr{Y}\left(X_{1}\right) \\
& =\sum_{s . t=p} \tau_{x}(s, t) \delta_{t} x . \mathscr{S}\left(\delta_{s}(t \triangleright x)\right) \\
& =\sum_{s . t=p} \tau_{x}(s, t) \tau_{s \succ(t \triangleright x)}\left(s, s^{R}\right)^{-1} \sigma_{s^{R}}\left(s \succ(t \triangleright x),(s \succ(t \triangleright x))^{-1}\right)^{-1} \\
& \times \delta_{t} x . \delta_{(s \triangleleft(t \triangleright x))^{R}}(s \succ(t \triangleright x))^{-1} \\
& =\sum_{s . t=p} \tau_{x}(s, t) \tau_{s \succ(t \triangleright x)}\left(s, s^{R}\right)^{-1} \sigma_{s^{R}}\left(s \succ(t \triangleright x),(s \succ(t \triangleright x))^{-1}\right)^{-1} \\
& \times \delta_{t \triangleleft x,(s \triangleleft(t \triangleright x))^{R}} \sigma_{t}\left(x,(s \succ(t \triangleright x))^{-1}\right) \delta_{t} x(s \succ(t \triangleright x))^{-1} .
\end{aligned}
$$

By Lemma 4.9(iv), we have $\delta_{t \triangleleft x,(s \triangleleft(t \triangleright x))^{R}}=\delta_{s, t^{L}}$. Hence, using Lemma 4.11(i), the last expression equals

$$
\begin{aligned}
\delta_{p, e} \sum_{s \in Q} \tau_{x}\left(s, s^{R}\right) \tau_{s \succ\left(s^{R} \triangleright x\right)}\left(s, s^{R}\right)^{-1} \sigma_{s^{R}}\left(s \succ\left(s^{R} \triangleright x\right),\left(s \succ\left(s^{R} \triangleright x\right)\right)^{-1}\right)^{-1} \\
\quad \times \sigma_{s^{R}}\left(x,\left(s \succ\left(s^{R} \triangleright x\right)\right)^{-1}\right) \delta_{s^{R}} x\left(s \succ\left(s^{R} \triangleright x\right)\right)^{-1} \\
=\delta_{p, e} \sum_{s \in Q} \delta_{s^{R}}=\delta_{p, e} 1=\delta_{p, e} \alpha .
\end{aligned}
$$


This proves the right-hand identity in (2-2). We now compute

$$
\begin{aligned}
& X_{1} \circ \beta \circ \mathscr{Y}\left(X_{2}\right) \\
& =\mathscr{Y}\left(X_{2}\right) \cdot \beta \cdot X_{1} \\
& =\sum_{s . t=p} \tau_{x}(s, t) \mathscr{S}\left(\delta_{t} x\right) \cdot \beta . \delta_{s}(t \triangleright x) \\
& =\sum_{s . t=p} \sum_{q} \omega\left(q, q^{-1}, q\right)^{-1} \tau_{x}(s, t) \mathscr{Y}\left(\delta_{t} x\right) . \delta_{q} \theta\left(q^{L}, q\right)^{-1} . \delta_{s}(t \triangleright x) \\
& =\sum_{s . t=p} \sum_{q} \omega\left(q, q^{-1}, q\right)^{-1} \tau_{x}(s, t) \tau_{t \succ x}\left(t, t^{R}\right)^{-1} \sigma_{t^{R}}\left(t \succ x,(t \succ x)^{-1}\right)^{-1} \\
& \times \delta_{(t \triangleleft x)^{R}}(t \succ x)^{-1} . \delta_{q} \theta\left(q^{L}, q\right)^{-1} . \delta_{S}(t \triangleright x) \\
& =\sum_{s . t=p} \sum_{q} \omega\left(q, q^{-1}, q\right)^{-1} \tau_{x}(s, t) \tau_{t \succ x}\left(t, t^{R}\right)^{-1} \sigma_{t^{R}}\left(t \succ x,(t \succ x)^{-1}\right)^{-1} \\
& \times \delta_{(t \triangleleft x)^{R} \triangleleft(t \succ x)^{-1}, q} \sigma_{(t \triangleleft x)^{R}}\left((t \succ x)^{-1}, \theta\left(q^{L}, q\right)^{-1}\right) \\
& \times \delta_{(t \triangleleft x)^{R}}(t \succ x)^{-1} \theta\left(q^{L}, q\right)^{-1} \delta_{s}(t \triangleright x) .
\end{aligned}
$$

By Lemma 4.11(ii), this equals

$$
\begin{aligned}
\sum_{s . t=p} \omega\left(t^{R},\left(t^{R}\right)^{-1}, t^{R}\right)^{-1} \tau_{x}(s, t) \tau_{t \succ x}\left(t, t^{R}\right)^{-1} \sigma_{t^{R}}\left(t \succ x,(t \succ x)^{-1}\right)^{-1} \\
\quad \times \sigma_{(t \triangleleft x)^{R}}\left((t \succ x)^{-1}, \theta\left(t, t^{R}\right)^{-1}\right) \delta_{(t \triangleleft x)^{R}}(t \succ x)^{-1} \theta\left(t, t^{R}\right)^{-1} \delta_{s}(t \triangleright x),
\end{aligned}
$$

or again

$$
\begin{aligned}
\sum_{s . t=p} \omega\left(t^{R},\left(t^{R}\right)^{-1}, t^{R}\right)^{-1} & \tau_{x}(s, t) \tau_{t \succ x}\left(t, t^{R}\right)^{-1} \sigma_{t^{R}}\left(t \succ x,(t \succ x)^{-1}\right)^{-1} \\
\times \sigma_{(t \triangleleft x)^{R}}((t & \left.\succ x)^{-1}, \theta\left(t, t^{R}\right)^{-1}\right) \sigma_{(t \triangleleft x)^{R}}\left((t \succ x)^{-1} \theta\left(t, t^{R}\right)^{-1}, t \triangleright x\right) \\
\times & \delta_{(t \triangleleft x)^{R} \triangleleft(t \succ x)^{-1} \theta\left(t, t^{R}\right)^{-1}, s} \delta_{(t \triangleleft x)^{R}}(t \succ x)^{-1} \theta\left(t, t^{R}\right)^{-1}(t \triangleright x),
\end{aligned}
$$

which in turn, by Lemma 4.11(ii) and Definition 4.10, is equal to

$$
\begin{aligned}
\delta_{p, e} \sum_{t} \omega\left(t^{R},\left(t^{R}\right)^{-1}, t^{R}\right)^{-1} \tau_{x}\left(t^{L}, t\right) \tau_{t \succ x}\left(t, t^{R}\right)^{-1} & \\
& \times \sigma_{t^{R}}\left(t \succ x,(t \succ x)^{-1}\right)^{-1} \sigma_{(t \triangleleft x)^{R}}\left((t \succ x)^{-1}, \theta\left(t, t^{R}\right)^{-1}\right) \\
& \times \sigma_{(t \triangleleft x)^{R}}\left((t \succ x)^{-1} \theta\left(t, t^{R}\right)^{-1}, t \triangleright x\right) \delta_{(t \triangleleft x)^{R}} \theta\left(t \triangleleft x,(t \triangleleft x)^{R}\right)^{-1} .
\end{aligned}
$$

We now evaluate the product

$\sigma_{t^{R}}\left(t \succ x,(t \succ x)^{-1}\right)^{-1} \sigma_{(t \triangleleft x)^{R}}\left((t \succ x)^{-1}, \theta\left(t, t^{R}\right)^{-1}\right) \sigma_{(t \triangleleft x)^{R}}\left((t \succ x)^{-1} \theta\left(t, t^{R}\right)^{-1}, t \triangleright x\right)$

appearing in the last sum. We use the cocycle condition (4-5) and Lemma 4.9 and obtain for it 


$$
\begin{aligned}
& \sigma_{t^{R}}\left(\theta\left(t, t^{R}\right)^{-1}, t \triangleright x\right) \sigma_{t^{R}}\left(t \succ x, \theta\left(t \triangleleft x,(t \triangleleft x)^{R}\right)^{-1}\right)^{-1} \\
& =\omega\left(t^{R}, \theta\left(t, t^{R}\right)^{-1}, t \triangleright x\right) \\
& \quad \times \omega\left(t^{R}, \theta\left(t, t^{R}\right)^{-1}(t \triangleright x) \theta\left(t \triangleleft x,(t \triangleleft x)^{R}\right), \theta\left(t \triangleleft x,(t \triangleleft x)^{R}\right)^{-1}\right)^{-1} \\
& =\omega\left(t^{R}, \theta\left(t, t^{R}\right)^{-1}, t \triangleright x\right) \omega\left((t \triangleleft x)^{L}, \theta\left(t \triangleleft x,(t \triangleleft x)^{R}\right), \theta\left(t \triangleleft x,(t \triangleleft x)^{R}\right)^{-1}\right)^{-1} \\
& \quad \times \omega\left(t^{R}, \theta\left(t, t^{R}\right)^{-1}(t \triangleright x), \theta\left(t \triangleleft x,(t \triangleleft x)^{R}\right)\right), \\
& =\omega\left((t \triangleleft x)^{L}, \theta\left(t \triangleleft x,(t \triangleleft x)^{R}\right), \theta\left(t \triangleleft x,(t \triangleleft x)^{R}\right)^{-1}\right)^{-1} \\
& \quad \times \omega\left(t^{L}, t \triangleright x, \theta\left(t \triangleleft x,(t \triangleleft x)^{R}\right)\right) \omega\left(t^{R}, \theta\left(t, t^{R}\right)^{-1},(t \triangleright x) \theta\left(t \triangleleft x,(t \triangleleft x)^{R}\right)\right) \\
& =\omega\left((t \triangleleft x)^{L}, \theta\left(t \triangleleft x,(t \triangleleft x)^{R}\right), \theta\left(t \triangleleft x,(t \triangleleft x)^{R}\right)^{-1}\right)^{-1} \\
& \quad \times \omega\left((t \triangleleft x)^{L}, t \triangleleft x,(t \triangleleft x)^{R}\right)^{-1} \omega\left(t^{L}, t, x\right)^{-1} \omega\left(t^{L}, t, x(t \triangleleft x)^{R}\right) \\
& \quad \times \omega\left(t^{R}, \theta\left(t, t^{R}\right)^{-1},(t \triangleright x) \theta\left(t \triangleleft x,(t \triangleleft x)^{R}\right)\right),
\end{aligned}
$$

where we have used the definition of $\sigma$ (4-4), the cocycle condition on $\omega$, and Lemma 4.3(iii).

On the other hand,

$$
\begin{aligned}
& \omega\left((t \triangleleft x)^{L}, \theta\left(t \triangleleft x,(t \triangleleft x)^{R}\right), \theta\left(t \triangleleft x,(t \triangleleft x)^{R}\right)^{-1}\right)^{-1} \omega\left((t \triangleleft x)^{L}, t \triangleleft x,(t \triangleleft x)^{R}\right)^{-1} \\
& =\omega\left((t \triangleleft x)^{R}, \theta\left(t \triangleleft x,(t \triangleleft x)^{R}\right)^{-1},(t \triangleleft x)(t \triangleleft x)^{R}\right)^{-1} \omega\left((t \triangleleft x)^{L}, t \triangleleft x,(t \triangleleft x)^{R}\right)^{-1} \\
& =\omega\left((t \triangleleft x)^{-1}, t \triangleleft x,(t \triangleleft x)^{R}\right) \omega\left((t \triangleleft x)^{R},\left((t \triangleleft x)^{R}\right)^{-1},(t \triangleleft x)^{R}\right)^{-1} \\
& \quad \times \omega\left((t \triangleleft x)^{L}, t \triangleleft x,(t \triangleleft x)^{R}\right)^{-1} \\
& =\omega\left((t \triangleleft x)^{R},\left((t \triangleleft x)^{R}\right)^{-1},(t \triangleleft x)^{R}\right)^{-1} .
\end{aligned}
$$

\section{Therefore}

$$
\begin{gathered}
\omega\left(t^{R},\left(t^{R}\right)^{-1}, t^{R}\right)^{-1} \tau_{x}\left(t^{L}, t\right) \tau_{t \succ x}\left(t, t^{R}\right)^{-1} \sigma_{t^{R}}\left(t \succ x,(t \succ x)^{-1}\right)^{-1} \\
\times \sigma_{(t \triangleleft x)^{R}}\left((t \succ x)^{-1}, \theta\left(t, t^{R}\right)^{-1}\right) \sigma_{(t \triangleleft x)^{R}\left((t \succ x)^{-1} \theta\left(t, t^{R}\right)^{-1}, t \triangleright x\right)},{ }^{-1} \omega\left((t \triangleleft x)^{R},\left((t \triangleleft x)^{R}\right)^{-1},(t \triangleleft x)^{R}\right)^{-1} \\
=\omega\left(t^{R},\left(t^{R}\right)^{-1}, t^{R}\right)^{-1} \omega\left(t, t^{R}, t \succ x\right)^{-1} \omega\left(t^{-1}, t, x(t \triangleleft x)^{R}\right) \omega\left(t^{R}, \theta\left(t, t^{R}\right)^{-1},(t \triangleright x) \theta\left(t \triangleleft x,(t \triangleleft x)^{R}\right)\right) \\
\left.\times \omega\left(t \triangleleft x,(t \triangleleft x)^{R}\right)\right)^{-1} \\
=\omega\left(t^{R},\left(t^{R}\right)^{-1}, t^{R}\right)^{-1} \omega\left(t, t^{R} \theta\left(t, t^{R}\right)^{-1},(t \triangleright x) \theta(t \triangleleft x)^{R}\right)^{-1},(t \triangleleft x)^{-1} \\
\times \omega\left(t, t^{R}, \theta\left(t, t^{R}\right)^{-1}\right)^{-1} \omega\left((t \triangleleft x)^{R},\left((t \triangleleft x)^{R}\right)^{R}\right) \\
=\omega\left(t^{R},\left(t^{R}\right)^{-1}, t^{R}\right)^{-1} \omega\left(t, t^{-1}, t x(t \triangleleft x)^{R}\right)^{-1} \omega\left(t, t^{R}, \theta\left(t, t^{R}\right)^{-1}\right)^{-1}, x(t \triangleleft x)^{R} \\
\quad \times \omega\left((t \triangleleft x)^{R},\left((t \triangleleft x)^{R}\right)^{-1},(t \triangleleft x)^{R}\right)^{-1} \omega\left(t^{-1}, t, x(t \triangleleft x)^{R}\right) \\
=\omega\left(t^{R},\left(t^{R}\right)^{-1}, t^{R}\right)^{-1} \omega\left(t, t^{-1}, t\right)^{-1} \omega\left(t^{-1}, t, x(t \triangleleft x)^{R}\right)^{-1} \omega\left(t, t^{R}, \theta\left(t, t^{R}\right)^{-1}\right)^{-1} \\
\quad \times \omega\left((t \triangleleft x)^{R},\left((t \triangleleft x)^{R}\right)^{-1},(t \triangleleft x)^{R}\right)^{-1} \omega\left(t^{L}, t, x(t \triangleleft x)^{R}\right) \\
=\omega\left(t^{R},\left(t^{R}\right)^{-1}, t^{R}\right)^{-1} \omega\left(t, t^{R} \theta\left(t, t^{R}\right)^{-1}, t\right)^{-1} \omega\left(t, t^{R}, \theta\left(t, t^{R}\right)^{-1}\right)^{-1} \\
\quad \times \omega\left((t \triangleleft x)^{R},\left((t \triangleleft x)^{R}\right)^{-1},(t \triangleleft x)^{R}\right)^{-1}
\end{gathered}
$$




$$
\begin{aligned}
& =\left(\omega\left(t^{R},\left(t^{R}\right)^{-1}, t^{R}\right) \omega\left(t, t^{R}, \theta\left(t, t^{R}\right)^{-1} t\right) \omega\left((t \triangleleft x)^{R},\left((t \triangleleft x)^{R}\right)^{-1},(t \triangleleft x)^{R}\right)\right)^{-1} \\
& =\omega\left(t^{R},\left(t^{R}\right)^{-1}, t^{R}\right)^{-1} \\
& \quad \times \omega\left(\left(t^{R}\right)^{-1}, t^{R},\left(t^{R}\right)^{-1}\right)^{-1} \omega\left((t \triangleleft x)^{R},\left((t \triangleleft x)^{R}\right)^{-1},(t \triangleleft x)^{R}\right)^{-1} \\
& \quad \quad \times \quad\left((t \triangleleft x)^{R},\left((t \triangleleft x)^{R}\right)^{-1},(t \triangleleft x)^{R}\right)^{-1} .
\end{aligned}
$$

Hence we get

$$
\begin{aligned}
X_{1} \circ \beta \circ \mathscr{S}\left(X_{2}\right) & =\delta_{p, e} \sum_{t} \omega\left((t \triangleleft x)^{R},\left((t \triangleleft x)^{R}\right)^{-1},(t \triangleleft x)^{R}\right)^{-1} \delta_{(t \triangleleft x)^{R}} \theta\left(t \triangleleft x,(t \triangleleft x)^{R}\right)^{-1} \\
& =\delta_{p, e} \beta,
\end{aligned}
$$

which gives the right-hand identity in (2-2).

The proof of conditions (2-3) is straightforward, using the properties listed in Lemma 4.9 and the cocycle conditions. This finishes the proof of the theorem.

\section{Frobenius-Schur indicators for $\mathscr{C}(G, \omega, F, 1)$}

Let $G$ be a finite group, $F \subseteq G$ a subgroup, and $\omega: G \times G \times G \rightarrow k^{\times}$a 3-cocycle subject to the normalization conditions (4-2) and (4-3). We keep the notation of the previous sections for the skeleton maps $\triangleright, \triangleleft, \theta, \sigma$ and $\tau$.

We have a $k$-linear monoidal equivalence $\operatorname{Rep}\left(A^{\mathrm{op}}, \Phi\right) \sim \mathscr{C}(G, F, \omega, 1)$, where $\left(A^{\mathrm{op}}, \Phi\right)$ is the quasi-Hopf algebra attached to the data $(G, F, \omega)$ in Section 4. Our aim in this section is to give an explicit description of the canonical central element $v_{A^{\text {op }}} \in A^{\text {op }}$ and then of the Frobenius-Schur indicators for the quasi-Hopf algebra $A^{\mathrm{op}}$. It follows from gauge invariance of the Frobenius-Schur indicators that these depend only on the fusion category $\mathscr{C}(G, F, \omega, 1)$.

Let $\Lambda_{0}:=|F|^{-1} \sum_{x \in F} x \in k F$ be the normalized integral. The normalized two-sided integral $\Lambda \in A^{\text {op }}$ has the form

$$
\Lambda=\delta_{e} \Lambda_{0}=|F|^{-1} \sum_{x \in F} \delta_{e} x
$$

Proposition 5.1. The element $\beta$ is invertible with inverse

$$
\beta^{-1}=\sum_{p \in Q} \omega\left(p^{L}, p, p^{R}\right) \delta_{p^{L}} \theta\left(p, p^{R}\right)
$$

In addition, $\mathscr{S}(\beta)=\beta^{-1}$.

Proof. It is not difficult to check that the expression

$$
\sum_{p \in Q} \omega\left(p^{R},\left(p^{R}\right)^{-1}, p^{R}\right) \sigma_{p^{R}}\left(\theta\left(p, p^{R}\right)^{-1}, \theta\left(p, p^{R}\right)\right)^{-1} \delta_{p^{L}} \theta\left(p, p^{R}\right)
$$


defines an inverse for $\beta$. We claim that

$$
\omega\left(p^{R},\left(p^{R}\right)^{-1}, p^{R}\right) \sigma_{p^{R}}\left(\theta\left(p, p^{R}\right)^{-1}, \theta\left(p, p^{R}\right)\right)^{-1}=\omega\left(p^{L}, p, p^{R}\right),
$$

for all $p \in Q$. This will imply the claimed expression for $\beta^{-1}$.

Letting $q=p^{R}$, the equality of two lines above is equivalent to

$$
\omega\left(q, q^{-1}, q\right) \sigma_{q}\left(\theta\left(q^{L}, q\right)^{-1}, \theta\left(q^{L}, q\right)\right)^{-1}=\omega\left(q \triangleleft \theta\left(q^{L}, q\right)^{-1}, q^{L}, q\right) .
$$

To establish this latter equality, we note that $\theta\left(q^{L}, q\right)=q^{L} q \in F$, for all $q \in Q$. Then, applying the cocycle and normalization conditions on $\omega$, we get

$$
\begin{aligned}
\omega\left(q \triangleleft \theta\left(q^{L}, q\right)^{-1}, q^{L}, q\right) & =\omega\left(q, q^{-1}, q\right) \omega\left(q, \theta\left(q^{L}, q\right)^{-1}, q^{L} q\right)^{-1} \\
& =\omega\left(q, q^{-1}, q\right) \omega\left(q, \theta\left(q^{L}, q\right)^{-1}, \theta\left(q^{L}, q\right)\right)^{-1},
\end{aligned}
$$

proving the claim.

Next, using Lemma 4.11(iv), we get $\mathscr{S}\left(\delta_{p} \theta\left(p^{L}, p\right)^{-1}\right)=\tau_{\theta\left(p, p^{R}\right)^{-1}}\left(p, p^{R}\right)^{-1} \sigma_{p^{R}}\left(\theta\left(p, p^{R}\right)^{-1}, \theta\left(p, p^{R}\right)\right)^{-1} \delta_{p^{L}} \theta\left(p, p^{R}\right)$.

We now compute

$$
\begin{aligned}
\tau_{\theta\left(p, p^{R}\right)^{-1}}\left(p, p^{R}\right) & =\omega\left(p, p^{R},\left(p p^{R}\right)^{-1}\right) \\
& =\omega\left(p p^{R},\left(p^{R}\right)^{-1}, p^{-1}\right)^{-1} \omega\left(p, p^{R},\left(p^{R}\right)^{-1}\right) \omega\left(p^{R},\left(p^{R}\right)^{-1}, p^{-1}\right) \\
& =\omega\left(p, p^{R},\left(p^{R}\right)^{-1}\right) \omega\left(p^{R},\left(p^{R}\right)^{-1}, p^{-1}\right),
\end{aligned}
$$

because $p p^{R} \in F$. Similarly,

$$
\begin{aligned}
& \sigma_{p^{R}}\left(\theta\left(p, p^{R}\right)^{-1}, \theta\left(p, p^{R}\right)\right) \\
&=\omega\left(p^{R},\left(p^{R}\right)^{-1} p^{-1}, p p^{R}\right) \\
&=\omega\left(p^{R},\left(p^{R}\right)^{-1}, p^{-1}\right)^{-1} \omega\left(\left(p^{R}\right)^{-1}, p^{-1}, p p^{R}\right)^{-1} \omega\left(p^{R},\left(p^{R}\right)^{-1}, p^{R}\right) \\
&=\omega\left(p^{R},\left(p^{R}\right)^{-1}, p^{R}\right) \omega\left(p, p^{-1},\left(p p^{R}\right)\right)^{-1} .
\end{aligned}
$$

Hence

$$
\begin{aligned}
\tau_{\theta\left(p, p^{R}\right)^{-1}}\left(p, p^{R}\right) \sigma_{p^{R}}\left(\theta\left(p, p^{R}\right)^{-1}, \theta\left(p, p^{R}\right)\right) & =\omega\left(p, p^{-1}, p\right)^{-1} \omega\left(p^{-1}, p, p^{R}\right)^{-1} \\
& =\omega\left(p, p^{-1}, p\right)^{-1} \omega\left(p^{L}, p, p^{R}\right)^{-1} .
\end{aligned}
$$

Thus,

$$
\begin{aligned}
\mathscr{S}(\beta) & =\sum_{p} \omega\left(p^{-1}, p, p^{-1}\right) \mathscr{Y}\left(\delta_{p} \theta\left(p^{L}, p\right)^{-1}\right) \\
& =\sum_{p} \omega\left(p^{L}, p, p^{R}\right) \delta_{p^{L}} \theta\left(p, p^{R}\right)=\beta^{-1} .
\end{aligned}
$$


Theorem 5.2. The canonical central element $v_{A^{\text {op }}}$ is given by

Moreover,

$$
v_{A^{\mathrm{op}}}=|F|^{-1} \sum_{(q \triangleleft x) \cdot q=e} \omega(x q, x q, x q) \delta_{q}(x q)^{2} .
$$

$$
\begin{aligned}
v_{A^{\mathrm{op}}}=|F|^{-1} \sum_{(q \triangleleft x) . q=e} \tau_{x}(q \triangleleft x, q) \sigma_{q}(x, q \triangleright x) \omega & \left((q \triangleleft x)^{L}, q \triangleleft x, q\right) \\
& \times \sigma_{q}(x(q \triangleright x),(q \triangleleft x) q) \delta_{q}(x q)^{2} .
\end{aligned}
$$

Proof. Since the element $\beta$ corresponding to the quasi-antipode of $A^{\text {op }}$ is invertible and $\alpha=1$, we have $v_{A^{\text {op }}}=\left(\Lambda_{1} \circ \Lambda_{2}\right) \circ \beta^{-1}=\beta^{-1} \circ\left(\Lambda_{1} \circ \Lambda_{2}\right)$, and thus

$$
v_{A^{\mathrm{op}}}=\beta^{-1} \circ\left(\Lambda_{1} \circ \Lambda_{2}\right)=\left(\Lambda_{1} \circ \Lambda_{2}\right) \cdot \beta^{-1} .
$$

Using formula (5-1) for the integral $\Lambda$, we find

$$
\Delta(\Lambda)=\Lambda_{1} \otimes \Lambda_{2}=|F|^{-1} \sum_{x \in F} \sum_{q \in Q} \tau_{x}\left(q^{L}, q\right) \quad \delta_{q^{L}}(q \triangleright x) \otimes \delta_{q} x
$$

thus

$$
\Lambda_{1} \circ \Lambda_{2}=\Lambda_{2} \cdot \Lambda_{1}=|F|^{-1} \sum_{(q \triangleleft x) \cdot q=e} \tau_{x}(q \triangleleft x, q) \sigma_{q}(x, q \triangleright x) \delta_{q} x(q \triangleright x) .
$$

From these, we compute $\beta^{-1} \circ\left(\Lambda_{1} \circ \Lambda_{2}\right)$ and get the second expression for $v_{A^{\text {op }}}$. Now, for all $x \in F$ and $q \in Q$ such that $(q \triangleleft x) \cdot q=e$, we have

$$
\begin{aligned}
& \sigma_{q}(x, q \triangleright x) \sigma_{q}(x(q \triangleright x),(q \triangleleft x) q) \\
&=\sigma_{q \triangleleft x}(q \triangleright x,(q \triangleleft x) q) \sigma_{q}(x, q x q) \\
&=\omega(q \triangleleft x, q \triangleright x,(q \triangleleft x) q) \omega(q, x, q x q) \\
&=\omega(q \triangleleft x, q \triangleright x,(q \triangleleft x) q) \omega(q \triangleleft x, q, x q)^{-1} \omega(q, x q, x q) \\
&=\omega(q \triangleleft x, q \triangleright x,(q \triangleleft x) q) \omega(q \triangleleft x, q, x)^{-1} \omega(q x, q x, q)^{-1} \omega(q, x q, x q),
\end{aligned}
$$

the last equality because

$$
\omega(q \triangleleft x, q, x q)=\omega(q \triangleleft x, q, x) \omega(q \triangleleft x, q x, q)=\omega(q \triangleleft x, q, x) \omega(q x, q x, q) .
$$

On the other hand,

$$
\begin{aligned}
\omega\left((q \triangleleft x)^{L}, q \triangleleft x, q\right) & =\omega\left(q^{L} \triangleleft(q \triangleright x), q \triangleleft x, q\right) \\
& =\omega\left(q^{L}, q x, q\right) \omega\left(q^{L}, q \triangleright x,(q \triangleleft x) q\right)^{-1} \\
& =\omega(q \triangleleft x, q x, q) \omega(q \triangleleft x, q \triangleright x,(q \triangleleft x) q)^{-1} \\
& =\omega(q x, q x, q) \omega(q \triangleleft x, q \triangleright x,(q \triangleleft x) q)^{-1} .
\end{aligned}
$$


Therefore, as needed for (5-2),

$$
\begin{aligned}
\tau_{x}(q \triangleleft x, q) \sigma_{q}(x, q \triangleright x) \omega\left((q \triangleleft x)^{L}, q \triangleleft x, q\right) \sigma_{q}(x(q \triangleright x),(q \triangleleft x) q) \\
=\omega(q, x q, x q)=\omega(x q, x q, x q) .
\end{aligned}
$$

Remark 5.3. Computing instead $\left(\Lambda_{1} \circ \Lambda_{2}\right) \circ \beta^{-1}$, we get

$$
\begin{aligned}
v_{A^{\mathrm{op}}}=|F|^{-1} \sum_{(q \triangleleft x) \cdot q=e} \tau_{x}(q \triangleleft x, q) \sigma_{q}(x, q \triangleright x) \omega\left((q \triangleleft x)^{L}, q \triangleleft x, q\right) \\
\quad \times \omega\left((q \triangleleft x)^{L},(q \triangleleft x) q, x(q \triangleright x)\right) \delta_{(q \triangleleft x)^{L}}((q \triangleleft x)(q \triangleright x))^{2} .
\end{aligned}
$$

As a consequence of Theorem 5.2, we get the following expression for the Frobenius-Schur indicators. After suitable normalization, this expression allows to compute the Frobenius-Schur indicators for every group-theoretical category.

Corollary 5.4. Suppose $\chi$ is an irreducible character of $A^{\mathrm{op}}$. The Frobenius-Schur indicator of $\chi$ is given by

$$
\begin{aligned}
& \chi\left(v_{A^{\mathrm{op}}}\right)=|F|^{-1} \sum_{(q \triangleleft x) \cdot q=e} \omega(x q, x q, x q) \chi\left(\delta_{q}(x q)^{2}\right) \\
&=|F|^{-1} \sum_{(q \triangleleft x) \cdot q=e} \tau_{x}(q \triangleleft x, q) \sigma_{q}(x, q \triangleright x) \omega\left((q \triangleleft x)^{L}, q \triangleleft x, q\right) \\
& \times \sigma_{q}(x(q \triangleright x),(q \triangleleft x) q) \chi\left(\delta_{q}(x q)^{2}\right) .
\end{aligned}
$$

In the remainder of this section we aim to give an explicit description of the irreducible characters (and hence of the indicators) of $\mathscr{C}(G, \omega, F, 1)$ in terms of the groups $G$ and $F$.

As an algebra $A=k^{Q_{\#_{\sigma}}} k F$ is a crossed product (page 362). Hence the irreducible left $A$-modules can be described using Clifford theory.

On the other hand, to every left $A$-module $V$ one can associate the left $A^{\text {op }}$ module $V^{*}$, the action of $a \in A^{\text {op }}$ being the transpose of the action of $a \in A$ on $V$. This gives a bijective correspondence between (irreducible) left $A$-modules $V$ and (irreducible) left $A^{\mathrm{op}}$-modules. Moreover, this bijection preserves characters: $\chi_{V^{*}}=\chi_{V}$, for all finite-dimensional left $A$-module $V$.

Let $F^{p} \subseteq F$ denote the isotropy subgroup of $p \in Q$. Then the restriction of $\sigma_{p}$ defines a normalized 2-cocycle

$$
\sigma_{p}: F^{p} \times F^{p} \rightarrow k^{\times}
$$

Let $k_{\sigma_{p}} F^{p}$ denote the corresponding twisted group algebra.

The space of isomorphism classes of irreducible $A$-modules can be parametrized by the modules $V_{p, W}$, where

$$
V_{p, W}=\operatorname{Ind}_{k Q_{\#_{\sigma}} k F^{p}} p \otimes W=A \otimes_{k} Q_{\#_{\sigma} k F^{p}}(p \otimes W),
$$


where $p$ runs over a set of representatives of the action of $F$ on $Q$, and $W$ runs over a system of representatives of isomorphism classes of irreducible left $k_{\sigma_{p}} F^{p}$ modules. See [Kashina et al. 2002, Section 3].

There is a natural identification between $Q$ and the space $F \backslash G=\{F g: g \in G\}$ of left cosets of $F$ in $G$. Under this identification, the action $Q \times F \rightarrow Q$ corresponds to the natural action of $F$ on $F \backslash G$ by right multiplication: $F g . x=F(g x), g \in G$, $x \in F$.

This gives in turn a natural identification between the space of orbits of the action $Q \times F \rightarrow Q$ and the space $F \backslash G / F$ of double cosets of $F$ in $G$. Moreover, the isotropy subgroup of an element $p \in Q$ is $F^{p}=F \cap p^{-1} F p$. Hence we get

Proposition 5.5. The set of isomorphism classes of irreducible $A^{\mathrm{op}}$-modules is parametrized by the modules $U_{p, W}$, where

$$
U_{p, W}=V_{p, W}^{*}=\left(\operatorname{Ind}_{k Q_{\#_{\sigma}} k F^{p}} p \otimes W\right)^{*},
$$

where $p$ runs over a set of representatives of the double cosets of $F$ in $G, F^{p}=$ $F \cap p^{-1} F p$, and $W$ runs over a system of representatives of isomorphism classes of irreducible left $k_{\sigma_{p}} F^{p}$-modules.

The character of the irreducible $A^{\mathrm{op}}$-module $U_{p, W}$ is given by the formula

$$
\chi_{p, W}\left(\delta_{q} z\right)=\sum_{y^{-1} z y \in F^{p}} \delta_{p, q \triangleleft y} \sigma_{q}(z, y) \sigma_{q}\left(y, y^{-1} z y\right)^{-1} \chi_{W}\left(y^{-1} z y\right),
$$

where the sum is over all y running over a set of representatives of the right cosets of $F^{p}$ in $F$, and $\chi_{W}$ is the character of $W$.

Observe that $\operatorname{dim} U_{p, W}=\left[F: F \cap p^{-1} F p\right] \operatorname{dim} W$. So the proposition immediately implies that the dimensions of the irreducible modules of a group-theoretical quasi-Hopf algebra divide its dimension, i.e., that Kaplanksy's conjecture holds in this case.

Proof. We only need to prove the formula for the character. The character of $U_{p, W}$ coincides with the character of $V_{p, W}$. Let $Y$ be a set of representatives of the right cosets of $F^{p}$ in $F$. A basis of $V_{p, W}$ is given by $y \otimes p \otimes v$, where $(v)$ is a basis of $W$, and $y \in Y$.

For all $q \in Q, y, z \in F$, we have

$$
\begin{aligned}
\left(\delta_{q} z\right) \cdot y & =\sigma_{q}(z, y) \delta_{q} z y \\
& =\sigma_{q}(z, y) \delta_{q} y\left(y^{-1} z y\right) \\
& =\sigma_{q}(z, y) \sigma_{q}\left(y, y^{-1} z y\right)^{-1} y .\left(\delta_{q \triangleleft y} y^{-1} z y\right) .
\end{aligned}
$$


Hence, the action of $\delta_{q} z$ on this basis is

$$
\begin{aligned}
\left(\delta_{q} z\right) \cdot y \otimes p \otimes v & =\left(\delta_{q} z\right) \cdot y \otimes p \otimes v \\
& =\sigma_{q}(z, y) \sigma_{q}\left(y, y^{-1} z y\right)^{-1} y \cdot\left(\delta_{q \triangleleft y} y^{-1} z y\right) \otimes p \otimes v .
\end{aligned}
$$

Thus, in order to compute the trace of this action, we only need to consider those basis vectors $y \otimes p \otimes v$, for which $y^{-1} z y \in F^{p}$; and for such $y$, we have

$$
\begin{aligned}
\left(\delta_{q} z\right) . y \otimes p \otimes v & =\sigma_{q}(z, y) \sigma_{q}\left(y, y^{-1} z y\right)^{-1} y\left(\delta_{q \triangleleft y} y^{-1} z y\right) \otimes p \otimes v \\
& =\delta_{p, q \triangleleft y} \sigma_{q}(z, y) \sigma_{q}\left(y, y^{-1} z y\right)^{-1} y \otimes p \otimes\left(y^{-1} z y\right) . v .
\end{aligned}
$$

This implies the desired formula.

Remark 5.6. The parametrization in Proposition 5.5 yields the statement in [Ostrik 2002, Remark after Proposition 3.1], for the category $\mathscr{C}(G, \omega, F, 1)$. Indeed, the 2cocycle $\psi^{p}(x, y) \in Z^{2}\left(F^{p}, k^{\times}\right)$considered there coincides with our $\sigma_{p}\left(y^{-1}, x^{-1}\right)$; and this is cohomologous to $\sigma_{p}(x, y)$ via $d(\gamma)$, where $\gamma(x)=\sigma_{p}\left(x^{-1}, x\right), x \in F$.

\section{Examples}

We now discuss some special cases of the results in the last two sections.

Abelian extensions. Suppose that $G=F Q$ is an exact factorization of the group $G$; that is, $Q$ is a subgroup of $G$ and $(F, Q)$ is a matched pair of finite groups with the actions $\triangleright: Q \times F \rightarrow F, \triangleleft: Q \times F \rightarrow Q$. We refer the reader to [Masuoka $1999 ; 2002]$ for the main notions used here, and in particular for the study of the cohomology theory associated to the matched pair $(F, Q)$.

Fix a representative $(\tau, \sigma)$ of a class in $\operatorname{Opext}\left(k^{G}, k F\right)$; that is,

$$
\sigma: F \times F \rightarrow\left(k^{Q}\right)^{\times} \quad \text { and } \quad \tau: Q \times Q \rightarrow\left(k^{F}\right)^{\times}
$$

are normalized 2-cocycles subject to compatibility conditions. Then consider the 3-cocycle $\omega: G \times G \times G \rightarrow k^{\times}$given by

$$
\omega(\tau, \sigma)(x p, y q, z r)=\tau_{z}(p \triangleleft y, q) \sigma_{p}(y, q \triangleright z), \quad x, y, z \in F, p, q, r \in Q .
$$

The class of the cocycle $\omega=\omega(\tau, \sigma)$ is the image of the class of $(\tau, \sigma)$ in the Kac exact sequence [Schauenburg 2002a; Masuoka 2002].

It is not difficult to see that $\sigma$ and $\tau$ have the same meaning as in Section 4 (pages 362-363). Note that $\left.\omega\right|_{Q \times Q \times Q}=1$.

There is a bicrossed product Hopf algebra $A:=k^{G}{ } \#_{\sigma} k F$ corresponding to these data. As is well-known, this correspondence gives a bijection between the equivalence classes of Hopf algebra extensions

$$
1 \rightarrow k^{Q} \rightarrow A \rightarrow k F \rightarrow 1,
$$


and the abelian group $\operatorname{Opext}\left(k^{G}, k F\right)$. The Hopf algebra $A^{\text {op }}$ coincides with the (quasi-)Hopf algebra corresponding to $G, F$ and $\omega$, as in Theorem 4.4.

Applying Corollary 5.4, we find the following expression for the FrobeniusSchur indicators.

Proposition 6.1. Let $\chi$ be an irreducible character of $A^{\mathrm{op}}$. Then the FrobeniusSchur indicator of $\chi$ is given by

$$
\begin{aligned}
\chi\left(v_{A^{\mathrm{op}}}\right) & =|F|^{-1} \sum_{q \triangleleft x=q^{-1}} \tau_{x}\left(q^{-1}, q\right) \sigma_{q}(x, q \triangleright x) \quad \chi\left(\delta_{q} x(q \triangleright x)\right) \\
& =|F|^{-1} \sum_{q \triangleleft x=q^{-1}} \tau_{x}\left(q^{-1}, q\right) \sigma_{q}(x, q x q) \chi\left(\delta_{q}(x q)^{2}\right) .
\end{aligned}
$$

This formula coincides with the expression found in [Kashina et al. 2002], where the Frobenius-Schur indicators of cocentral abelian extensions are computed, i.e., extensions giving rise to the trivial action $\triangleright: F \times Q \rightarrow Q$. Corollary 5.4 gives also an alternative expression in terms of the 3-cocycle $\omega$ attached to $\sigma$ and $\tau$ via the Kac exact sequence.

Twisted quantum doubles. Let $G$ be a finite group and let $\omega$ be 3-cocycle on $G$. Consider the Dijkgraaf-Pasquier-Roche quasi-Hopf algebra $D^{\omega} G$, also called the twisted quantum double of $G$ [Dijkgraaf et al. 1991]. By the results in [Natale 2003], a semisimple quasi-Hopf algebra $H$ is group-theoretical if and only if its quantum double is gauge equivalent to a quasi-Hopf algebra $D^{\omega} G$. The FrobeniusSchur indicators for $D^{\omega} G$ have been computed in [Mason and $\mathrm{Ng} 2005$ ], and seen to coincide in this case with the indicators introduced by Bantay [1997].

It is shown in [Ostrik 2002] that the category $\operatorname{Rep} D^{\omega} G$ is equivalent to $\mathscr{C}(G \times$ $G, \widetilde{\omega}, \Delta(G), 1)$, where $\Delta(G) \simeq G$ is the diagonal subgroup of $G \times G$, and $\widetilde{\omega}$ is the 3 -cocycle on $G \times G$ given by $\widetilde{\omega}=p_{1}^{*} \omega\left(p_{2}^{*} \omega\right)^{-1}$; that is,

$$
\widetilde{\omega}\left(\left(a_{1}, a_{2}\right),\left(b_{1}, b_{2}\right),\left(c_{1}, c_{2}\right)\right)=\omega\left(a_{1}, b_{1}, c_{1}\right) \omega\left(a_{2}, b_{2}, c_{2}\right)^{-1}, \quad a_{i}, b_{i} \in G .
$$

Thus Corollary 5.4 gives an alternative formula for the Frobenius-Schur indicators of $D^{\omega} G$ in terms of an appropriate normalization of the 3-cocycle $\widetilde{\omega}$.

\section{Acknowledgements}

This work was began during a postdoctoral stay at the Department of Mathematics of the École Normale Supérieure, Paris. The author is grateful to Marc Rosso for his kind hospitality. She also thanks Peter Schauenburg for helpful comments about Theorem 4.12. Special thanks go to Susan Montgomery for interesting discussions and to the Department of Mathematics of the University of Southern California for their support and warm hospitality during the author's visit in October 2003. 


\section{References}

[Bantay 1997] P. Bantay, "The Frobenius-Schur indicator in conformal field theory", Phys. Lett. B 394:1-2 (1997), 87-88. MR 98c:81195 Zbl 0925.81331

[Beggs 2003] E. Beggs, "Making non-trivially associated tensor categories from left coset representatives”, J. Pure Appl. Algebra 177:1 (2003), 5-41. MR 2004e:18010 Zbl 1037.18004

[Beggs and Al-Shomrani 2003] E. Beggs and M. Al-Shomrani, "Making non-trivially associated modular categories from left coset representatives", preprint, 2003. math.QA/0303058

[Dijkgraaf et al. 1991] R. Dijkgraaf, V. Pasquier, and P. Roche, "Quasi-quantum groups related to orbifold models", pp. 375-383 in Modern quantum field theory (Bombay, 1990), edited by S. Das et al., World Sci. Publishing, River Edge, NJ, 1991. MR 1237434 Zbl 0854.17012

[Drinfeld 1989] V. G. Drinfel'd, “Quasi-Hopf algebras”, Algebra i Analiz 1:6 (1989), 114-148. In Russian; translated in Leningrad Math. J. 1:6 (1990), 1419-1457. MR 91b:17016

[Etingof and Gelaki 2002] P. Etingof and S. Gelaki, "On families of triangular Hopf algebras", Int. Math. Res. Not. 14 (2002), 757-768. MR 2002m:16036 Zbl 0998.16028

[Etingof et al. 2002] P. Etingof, D. Nikshych, and V. Ostrik, "On fusion categories", preprint, 2002. math.QA/0203060

[Fuchs et al. 1999] J. Fuchs, , A. C. Ganchev, K. Szlachányi, and P. Vecsernyés, " $S_{4}$ symmetry of $6 j$ symbols and Frobenius-Schur indicators in rigid monoidal $C^{*}$ categories", J. Math. Phys. 40:1 (1999), 408-426. MR 99k:81111 Zbl 0986.81044

[Hausser and Nill 1999] F. Hausser and F. Nill, "Diagonal crossed products by duals of quasiquantum groups", Rev. Math. Phys. 11:5 (1999), 553-629. MR 2000d:81069 Zbl 1032.81020

[Kashina et al. 2002] Y. Kashina, G. Mason, and S. Montgomery, "Computing the Frobenius-Schur indicator for abelian extensions of Hopf algebras", J. Algebra 251:2 (2002), 888-913. MR 2003f: 16061 Zbl 1012.16040

[Linchenko and Montgomery 2000] V. Linchenko and S. Montgomery, "A Frobenius-Schur theorem for Hopf algebras", Algebras and Representation Theory 3:4 (2000), 347-355. MR 2001k:16073 Zbl 0971.16018

[Mason and Ng 2005] G. Mason and S.-H. Ng, "Central invariants and Frobenius-Schur indicators for semisimple quasi-Hopf algebras", Adv. Math. 190:1 (2005), 161-195. MR 2005h:16066 Zbl 02126447

[Masuoka 1999] A. Masuoka, "Extensions of Hopf algebras", Trabajo de matemática 41/99, FaMAF, Universidad Nacional de Córdoba (Argentina), 1999.

[Masuoka 2002] A. Masuoka, "Hopf algebra extensions and cohomology", pp. 167-209 in New directions in Hopf algebras, Math. Sci. Res. Inst. Publ. 43, Cambridge Univ. Press, Cambridge, 2002. MR 2003d:16050 Zbl 1011.16024

[Natale 2003] S. Natale, "On group theoretical Hopf algebras and exact factorizations of finite groups”, J. Algebra 270:1 (2003), 199-211. MR 2004k:16102 Zbl 1040.16027

[Ostrik 2002] V. Ostrik, "Boundary conditions for holomorphic orbifolds", 2002. math.QA/0202130

[Schauenburg 2002a] P. Schauenburg, "Hopf bimodules, coquasibialgebras, and an exact sequence of Kac", Adv. Math. 165:2 (2002), 194-263. MR 2003e:16052 Zbl 1006.16054

[Schauenburg 2002b] P. Schauenburg, "Two characterizations of finite quasi-Hopf algebras", 2002. math.QA/0207069

[Serre 1967] J.-P. Serre, Représentations linéaires des groupes finis, Hermann, Paris, 1967. MR 38 \#1190 Zbl 0189.02603 
[Tambara and Yamagami 1998] D. Tambara and S. Yamagami, "Tensor categories with fusion rules of self-duality for finite abelian groups”, J. Algebra 209:2 (1998), 692-707. MR 2000b:18013 Zbl 0923.46052

[Zhu 2001] Y. Zhu, "Hecke algebras and representation ring of Hopf algebras", pp. 219-227 in First International Congress of Chinese Mathematicians (Beijing, 1998), edited by Y. Lo and S.-T. Yau, AMS/IP Stud. Adv. Math. 20, Amer. Math. Soc., Providence, RI, 2001. MR 2002c:20011 Zbl 02084970

Received December 26, 2003. Revised October 5, 2004.

SONiA NATALE

FACUlTAd de MATEMÁticA, Astronomía y Física

UNIVERSIDAD NACIONAL DE CÓRDOBA

CIEM - CONICET

(5000) CIUDAD UNIVERSITARIA

CÓRDOBA

Argentina

natale@mate.uncor.edu

http://www.mate.uncor.edu/natale 